\title{
La fisiología vegetal en la botánica actual en México
}

\author{
ALFONSO LARQUÉ-SAAVEDRA \\ Instituto de Recursos Naturales, Progrnna de Botánica.Colegio de Postgraduados. Montecillos, Texcoco, \\ Miéxico CP 56230
}

\begin{abstract}
Resumen. A partir de 1980, las investigaciones en fisiología vegetal en México se han incrementado sustancialmente en comparación con lo realizado anteriormente, como resultado de: i) la interacción de investigadores mexicanos y de otros países en eventos científicos realizados en México, ii) su institucionalización al crearse el postgrado de esta disciplina, iii) el interés de las principales instituciones académicas de este país por apoyar su desarrollo y iv) el incremento de investigadores dedicados a esta disciplina. La mayoría de las investigaciones ha sido realizada en plantas superiores de interés agrícola originarias o introducidas en México, y en menor grado en comunidades naturales. Presentamos aquí algunas de las contribuciones científicas.
\end{abstract}

Abstract. Research in Plant Physiology has grown substantially in México since 1980 in comparison with previous years. The reasons for such increase are due to i) the interaction between mexican scientists and scientists from other countries in meetings carried out in Mexico, ii) the creation of a graduate programme in plant physiology, iii) the economic support of the main academic institutions to this discipline and iv) the increase in the number of scientists working in plant physiology. The research carried out in this field has been done mainly in native or introduced cultivated plants of agronomic importance. Some of the scientific contributions are presented.

\section{INTRODUCCIÓN}

La enorme diversidad de especies vegetales que existe en México representa un reto para cualquier estudioso de la botánica en general y por supuesto de la fisiología vegetal. En 1978, Rzedowski calculaba en trescientos mil el número de especies vasculares que hay en nuestro país. Hasta 1981, el $40 \%$ del territorio estaba cubierto por comunidades naturales con diferentes grados de perturbación; entre ellas el matorral ocupaba la mayor extensión (36\%) (Moreno-Casasola y Sánchez, 1990).

En México, los estudios de fisiología vegetal de esa inmensa riqueza vegetal son muy escasos, y no llegan a cubrir ni el $0.1 \%$ del total de las especies de las que Rzedowski toma nota. El mayor número de trabajos se ha realizado en especies, introducidas o no, de interés agrícola (Cuadro 1). De dichos estudios se extrae que el $60 \%$ se han realizado en plantas enteras, el $20 \%$ en explantes, el $20 \%$ en semillas y el $15 \%$ en organelos (mitocondrias, cloroplastos, núcleo, etc).

En orden de importancia, los campos de la fisiología vegetal son: fisiología de cultivos $30 \%$; fisiología del estrés $25 \%$; micropropagación y cultivo de tejidos $15 \%$; fisiología de la germinación $12 \%$; ecofisiología $10 \%$; nutrición mineral 5\%; fisiología de postcosecha 3\%. Esta clasificación se basa en el número de publicaciones.

De igual modo los procesos fisiológicos atendidos son: crecimiento y desarrollo $20 \%$; relaciones agua-planta $18 \%$; fotosíntesis (biofísica, bioquímica, planta entera) $18 \%$; germinación 16\%; translocación (fuente y demanda) 12\%; hormonas vegetales $8 \%$; mecanismos de protección (metabolitos secundarios) $6 \%$; nutrición mineral $5 \%$; otros (floración, respiración,etc) $4 \%$.

\section{EVENTOS Y PROYECTOS INTERNACIONALES}

A partir de 1980, el impulso de la investigación en fisiología vegetal que se da en México se debe, en gran medida, a los eventos internacionales llevados a cabo en este país. En julio de 1980 el Centro de Botánica del Colegio de Postgraduados llevó a cabo el curso teórico-práctico «El agua en las plantas y fotosíntesis», impartido por los profesores D. W. Davies de la Universidad de Lancaster, M. K. Garret, de Queen's University de Belfast y A. Larqué-Saavedra. Este curso fue el primero en su género, pues por primera vez en México se abordaron estos temas con instrumental moderno: analizador de gases en el infra-rojo, porómetros, cámaras psicrométricas, etc. Este instrumental fue presentado a jóvenes interesados en estos campos del conocimiento. Tras la experiencia, no menos de seis de los estudiantes continuaron su formación doctoral en diferentes universidades del mundo, y son ahora promotores de estudios relacionados con estos campos de la fisiología vegetal.

En septiembre del mismo año, el CONACYT favoreció la realización de un curso que coordinó el prof.D. O. Hall de la Universidad de Londres, y que fue auspiciado con fondos de la UNEP. Titulado «International training course on techniques in bioproductivity and photosynthesis», el curso fue dictado en el Centro de Investigación en Química Aplicada (CIQA), de Saltillo, y tuvo repercusiones muy positivas en el ambiente botánico de México, pues permitió describir y llevar al campo numerosas técnicas de fisiología vegetal hasta entonces sólo realizadas en el laboratorio. Gracias a este curso se supo del interés del profesorP. Nobel, de la Universidad de California, por las agavaceas. Desde 
entonces, Nobel mantiene una intensa interacción académica con grupos de investigadores de Yucatán (CICY), del Centro de Botánica del Colegio de Postgraduados, del CIQA y más recientemente con los de la Universidad de Guadalajara.

Cuadro 1. Principales especies vegetales sobre las que se han realizado estudios de fisiología vegetal en México.

\begin{tabular}{ll}
\hline Silvestres & Cultivadas \\
\hline Prosopis spp. & Phaseolus vulgaris \\
Solanum cardiophylum & Zea mays \\
Larrea tridenta & Triticum vulgare \\
Piper hispidum & Sorghum bicolor \\
Piper aequale & Solanum tuberosum \\
Urera caracasana & Lycopersicum sculentum \\
Guarea glabra & Vicia faba \\
Poulsenia armata & Opuntia spp. \\
Brosimum alicastrum & Capsicum annum \\
Opuntia pilifera & Cucurbita spp. \\
Neobuxbania tetetzo & Pisum sativum \\
Ferocactus & Helianthus annuus \\
Preseskia & Coffea spp. \\
Agave salmiana & Saccharum spp. \\
Agave spp. & Musa spp. \\
Phaseolus lunatus & Gossypium hirsutum \\
Phaseoulus coccineus & Persea americana \\
Pinus cembroides & Terminalia catappa \\
Schinus molle & Prunus domestica \\
Pinus spp. & Citrus spp. \\
Buteloua spp. & Cocus nucifera \\
Distichlis spp. & Prunus armeniaca \\
Pharthenium spp. & Prunus persica \\
Simmondsia spp. & Mangifera indica \\
Salix ssp. & Malus spp. \\
Distichlis spicata & Vitis spp. \\
Kochria spp. & Medicago sativa \\
& Oryza sativa \\
& Nicotiana tobaco \\
& Amaranthus spp. \\
& \\
& \\
&
\end{tabular}

En 1982, el grupo de ecología vegetal de la UNAM invitó a los profesores $\mathrm{H}$. Mooney de la Universidad de Stanford, C. Field y N. Chiarello de la Universidad de Utah y al profesor F. Bazzaz que actualmente está en la Universidad de Harvard, a realizar una investigación en la estación biológica de los Tuxtlas, sobre aspectos ecofisiológicos de Piper. Esta primera interacción dio origen, en 1983, a un taller y simposium internacional titulado «Fisiología ecológica de plantas tropicales» que se llevó a cabo en Oaxtepec, Morelos. En esta reunión participaron, además de los investigadores antes señalados: E. Medina de Venezuela, R. Robichaux, T. Givnish, N. Fitcher, J. Landsberg, C. Jordan, de los Estados Unidos y C. Vázquez, A. Orozco y L. Fanjul de México. Se desarrollaron temas sobre las diferentes formas de crecimiento, el balance de nutrientes, hídrico y de carbono. Fueron presentados y discutidos trabajos como «Whole plant physiological demographic and environmental components», que estimularon nuevas tendencias de investigación en todo el mundo. Como resultado de esta reunión, y de investigaciones posteriores producto de esta interacción se publicó un libro, varios artículos y se estimuló la formación de recursos humanos en esta área del conocimiento.

A partir de los tres eventos antes señalados, considero que se establecen las bases del estudio de la fisiolgía vegetal «moderna» de México. Posteriormente se han dictado otros cursos que han apoyado de manera sistemática el desarrollo de la disciplina (Cuadro 2).

\section{INVESTIGACIONES SOBRE VEGETACIÓN NATURAL}

En 1972, Gómez Pompa y colaboradores publicaron en la revista Science (177:762-765) un artículo que, considero, fué el que propició el inicio de estudios de fisiología vegetal en especies de las selvas de México. A partir de entonces el Dr. Carlos Vázquez, quien por cierto es uno de los coautores de dicho artículo, empezó a conformar un grupo de investigadores, dentro de los que destaca la Dra. Alma

Cuadro 2. Cursos internacionales de fisiolgía vegetal dictados en años recientes en México.

\begin{tabular}{|c|c|}
\hline Año & Tema \\
\hline 1989 & Aspectos básicos y de fisiología estomática \\
\hline 1989 & $\begin{array}{l}\text { Distribución de fotosintatos y relación fuente } \\
\text { y demanda }\end{array}$ \\
\hline 1989 & $\begin{array}{l}\text { Regulación del crecimiento y desarrollo de } \\
\text { árboles frutales tropicales }\end{array}$ \\
\hline 1991 & Transporte de iones en vegetales \\
\hline 1991 & Rutas de detección del ambiente en vegetales \\
\hline 1991 & $\begin{array}{l}\text { La ecofisiología vegetal como herramienta para } \\
\text { abordar estudios de conservación de recursos } \\
\text { genéticos }\end{array}$ \\
\hline 1992 & Avances metodológicos en fisiología vegetal \\
\hline
\end{tabular}

Responsable

E. Zeiger (U. California)

J.Daie (U. de Rutgers)

T. Davenport (U. de Florida)

S.M. Assman (U. de Harvard)

S.J. Roux (U. de Texas)

R. Orellana (Coord.) CICY

J. Ehleringer (U. Utah) 
Orozco, quienes han venido publicando numerosos artículos sobre germinación de semillas de especies vegetales típicas de ese tipo de vegetación (Vázquez-Yanes y Orozco-Segovia, 1993).

Sus descubrimientos del fotoblastismo de las semillas (el proceso de germinación depende de la existencia de la luz), son contribuciones a la literatura internacional. Destaca la información acerca de que especies heliófitas (plantas que requieren luz solar intensa para crecer) como Piperhispidumy Piperaequale, producen semillas fotoblásticas positivas, y que la temperatura es un factor que regula 0 modifica la respuesta fotoblástica como en el caso de Urera caracasana. Para esta especie ellos reportan que variaciones diarias de temperatura de $25^{\circ}$ a $35^{\circ} \mathrm{C}$ disminuyen el número de horas luz para germinar. Sin embargo, anotan que estas temperaturas no pueden sustituir completamente el requerimento de luz (Orozco Segovia et al., 1987).

Por otro lado, han demostrado que la luz es un factor muy importante dentro del proceso de regeneración de las selvas, debido a que su composición espectral es modificada por la perturbación de cobertura vegetal, señalando además que las semillas de Piper enterradas pierden su requerimiento de luz y germinan antes de un año (Orozco Segovia y Vázquez-Yanes, 1989). Sus datos reportan que la luz filtrada por las hojas muertas de especies como Guarea glabra, Poulsenia amrmata, Brosimum alicastrum, inhiben la germinación de semillas. Las hojas hidratadas de estas especies bloquean preferentemente el paso de longitudes de onda superiores a $600 \mathrm{~nm}$.

Otro tipo de vegetación natural que ha recibido atención son las especies de climas áridos y semiáridos, en investigaciones que han sido conducidas por el Dr. E. Ezcurra y su grupo de investigadores que han publicado sus resultados para especies como Larrea tridentata, conocida como gobernadora, diferentes especies de Opuntia, Neobuxbaumia tetetzo, Ferocactus y Preseskia (Ezcurra et al., 1992).

Sus resultados indican que los movimientos foliares en Larrea, son fundamentalmente para el éxito de la especie en condiciones de alta irradiación. Esos movimientos están influenciados en gran medida además por el estatus hídrico de la planta, llegando a anotar que aquellos foliolos con déficit hídrico se abren poco y la intercepción de la radiación directa se reduce en $24 \%$. En Opuntia pilifera también se han señalado aquellos aspectos básicos para que ocurra la mejor orientación de los cladodios, y para optimizar la eficiencia fotosintética sin someterse a condiciones críticas de altas temperaturas. En 1992 publicaron los cambios fotosintéticos que ocurren durante la ontogenia de especies de cuatro familias del desierto, y descubrieron que durante las primeras etapas del desarrrollo, las plántulas presentan un metabolismo C-3, y 10 o 20 semanas después de la germinación se comportan como plantas típicas CAM. Es decir, que al inicio de su desarrollo estas especies recurren al metabolismo fotosintético de sus ancestros (Altesor et al., 1992).

\section{INVESTIGACIONES EN PLANTAS CULTIVADAS}

La institucionalización del programa de postgrado en fisiología vegetal, en 1989, por el Colegio de Postgraduados, ha estimulado sustancialmente la investigación en este campo. Sin embargo, hay que resaltar que prácticamente todas las investigaciones han sido conducidas sobre plantas cultivadas, lo cual no es del todo satisfactorio. En el Cuadro 3 se anota la información relevante de los temas abordados.

CUADRO 3. Información relevante de las investigaciones realizadas en plantas cultivadas por el postgrado en fisiología vegetal.

\begin{tabular}{|c|c|c|c|}
\hline Tema & Cultivo & Año & Referencia \\
\hline Fotosíntesis & Frijol & 1992 & Delgado, 1992 \\
\hline \multirow[t]{2}{*}{ Frío } & Frijol & 1992 & Lagunes, 1992 \\
\hline & Toronja & 1993 & Aguilar, 1993 \\
\hline Competencia & Maíz-Simsia & 1992 & Uscanga, 1992 \\
\hline \multirow{2}{*}{$\begin{array}{l}\text { Metabolitos } \\
\text { y nutrición }\end{array}$} & Kochia & 1992 & Baltazar, 1992 \\
\hline & Zarzamora & 1994 & Montes, 1994 \\
\hline \multirow[t]{6}{*}{ Sequía } & Amaranthus & 1992 & Trejo, 1992 \\
\hline & Frijol & 1992 & Solarte, 1992 \\
\hline & Sorgo & 1992 & Montes, 1992 \\
\hline & Maíz & 1993 & Salinas, 1992 \\
\hline & Trigo & 1994 & Cortázar, 1994 \\
\hline & Chile & 1993 & SantosDíaz, 1993 \\
\hline \multicolumn{4}{|l|}{$\begin{array}{l}\text { Longevidad } \\
\text { y vigor de }\end{array}$} \\
\hline \multirow[t]{3}{*}{ semillas } & Sorgo & 1993 & Galeano, 993 \\
\hline & Maíz & 1993 & Cruz, 1993 \\
\hline & Frijol & 1993 & García, 1994 \\
\hline \multirow{3}{*}{$\begin{array}{l}\text { Fuente y } \\
\text { demanda }\end{array}$} & & & \\
\hline & Maíz & 1993 & Mendoza, 1993 \\
\hline & Jitomate & 1994 & Sánchez, 1994 \\
\hline Postcosecha & Tunas & 1994 & Sáenz, 1994 \\
\hline \multicolumn{4}{|l|}{ Producción } \\
\hline forzada & Naranja & 1994 & Almaguer, 1994 \\
\hline \multicolumn{4}{|l|}{ Fisiología } \\
\hline patológica & Clavel & 1994 & Vázquez, 1994 \\
\hline \multicolumn{4}{|l|}{ Fijación de } \\
\hline nitrógeno & Frijol & 1994 & Almaráz, 1994 \\
\hline
\end{tabular}

Considero conveniente referir, dentro de los estudios de fisiología vegetal de investigaciones en plantas cultivadas, el de la relación de los parámetros fisiológicos con el de productividad ò rendimiento de los cultivos. Sobre el particular quisiera referir los trabajos iniciados por Delgado y colaboradores en 1991, y de Rees y colaboradores en 1992, porque considero que aportan información novedosa. Ambos trabajos fueron realizados en trigo, el primero de ellos por mi proyecto conjunto entre el Centro Internacional de Mejoramiento de Maíz y Trigo y el Colegio de Postgraduados, que se estableció en condiciones de campo en Tlatizapán, Morelos. En éste se estudiaron la fotosíntesis, la conductancia estomática, el contenido de clorofila y la respiración nocturna de 16 genotipos de trigo, crecidos en estrés de altas 
temperaturas, y su relación con el rendimiento. Las determinaciones fueron hechas durante tres estadios fenológicos de las plantas a diferentes horas del día. Los resultados de dos experimentos independientes revelaron una correlación significativa entre la velocidad de fotosíntesis, conductancia estomática, temperatura de la hoja y contenido de clorofila, con el rendimiento del grano. La velocidad de fotosíntesis después de la antesis fue asociada con la pérdida de clorofila. No fueron encontradas diferencias en otros parámetros, como la respiración o la fluorescencia estimadas en las hojas in situ.

El segundo trabajo se llevó a cabo también con trigo en Ciudad Obregón, Sonora, en un estudio conjunto entre el Centro Internacional de Mejoramiento de Maíz y Trigo, la Universidad de California y el Colegio de Postgraduados. Se investigó en ocho variedades liberadas desde 1962 (que son representativas de alto rendimiento explicado por el incremento del índice de cosecha) si el rendimiento estaba correlacionado con la conductancia estomática y con la velocidad de fotosíntesis, estimándose éstas antes y después de la antesis. Las correlaciones entre rendimiento y conductancia fueron altamente significativas en ambos periódos fenólogicos. La fotosíntesis y el rendimiento también fueron significativos. Las correlaciones hechas entre estas mediciones con la acumulación de biomasa no fueron significativas, como tampoco lo fue la fluorescencia.

La trascendencia de los resultados de ambos experimentos resulta ser particularmente importante, pues se plantea que la apertura estomática está relacionada con el rendimiento del grano. El potencial de uso de esta variable fisiológica en fitomejoramiento de plantas cultivadas, representaría una buena contribución al conocimiento fisiológico básico con repercusiones prácticas.

Finalmente, quiero referirme a dos estudios realizados que quizá sean únicos en su género. El primero de ellos se refiere al efecto que tiene el ciclo lunar con la producción de materia seca, área foliar y concentración de nutrientes en maíz (Anguiano,1992). Se reporta que los ciclos lunares no afectaron significativamente las variables estudiadas; sin embargo, se anota que las fases de luna llena y nueva son las que tienen mayores efectos sobre las variables estudiadas. Estos interesantes datos requieren mayor cotejo, por la trascenidencia de lo encontrado.

El segundo estudio se refiere al efecto que tuvo el eclipse total de sol en México en 1992, y es acerca del intercambio gaseoso (Nava y Larqué-Saavedra, información personal). Los resultados del estudio hecho en frijol, señalan que el eclipse afectó significativamente la apertura estomática.

\section{COMENTARIOS FINALES}

Existen estudios de fisiología vegetal en plantas cultivadas que se han realizado en el umbral con la bioquímica, la biofísica y la biología molecular. Sobre el particular resaltan, entre otros, los estudios sobre la transducción de señales, la inducción de genes por el efecto de la luz y la importancia de las nodulinas (Larqué Saavedra y Rodríguez,1989; Simpson y Herrera-Estrella, 1992; Sánchez et al., 1991; Sánchez de Jimenez, 1993). Estos aportes requieren otro tipo de análisis más allá del interés del presente escrito, sin embargo han sido hechos en plantas cultivadas.

Debo reiterar el hecho de que los avances de la fisiología vegetal en México, a partir de 1980, se deben en gran medida a los proyectos de enseñanza e investigación entre diferentes instituciones nacionales, y en gran medida al estímulo recibido por investigadores de otros países que han participado en las reuniones y cursos internacionales organizados en México, así como a los proyectos conjuntos derivados de esta interacción, algunos de los cuales fueron anotadas en este artículo. Ojalá que este espíritu de crítica y análisis de los comprometidos con la fisiología vegetal se siga dando para proseguir enriqueciendo esta área del conocimiento en México.

\section{LITERATURA CITADA}

Aguilar LJ. 1993. Efecto de temperaturas de acondicionamiento en el control de daños por frío de frutos de toronja «Red blus» $y$ «Marsh Seedless». Tesis de Maestría, Colegio de Postgraduados, México.

Almaguer VG. 1994. Producción forzada en naranja (Citrus sinensis L. Osbeck). Tesis de doctorado. Colegio de Postgraduados. México.

Almaraz SJJ. 1994. Diferencias entre el frijol silvestre y domesticado en atributos morfofisiológicos y en su respuesta al almacenamiento inadecuado de la semilla. Tesis de Maestría, Colegio de Postgraduados, México.

Altesor A, Ezcurra E, Silva C. 1992. Changes in the photosynthetic metabolism during the early ontogeny of four cactus species. Acta Ecológica 13: 777-785.

Anguiano JJM. 1992. Evaluación experimental y registro del conocimiento empírico sobre la influencia de las fases lunares en maíz (Zea mays L.). Tesis de Licenciatura. Universidad Autónoma de Chapingo, México.

Baltazar BO. 1992. Acumulación de oxalatos y nitratos durante el desarrollo de Kochia scoparia L. Schrad. Tesis de Maestría. Colegio de Postgraduados, México.

Cortázar RM. 1994. Efectos fisiológicos de deficiencias hídricas en tres genotipos de trigo (Triticum aestivum L.). Tesis de Maestría, Colegio de Postgraduados, México.

Cruz GF.1993. El metabolismo del ADN y el vigor de semillas de maíz. Tesis de Maestría. Colegio de Postgraduados, México.

Delgado AA.1992. Metabolitos formados por la fijación de $14 \mathrm{CO}_{2}$, en la cavidad de la vaina de Phaseolus vulgaris L. Tesis de Maestría, Colegio de Postgraduados, México.

Ezcurra E, Arizaga VMC, Flores MA. 1992. Foliole movement and canopy architecture of Larrea tridentata (DC.) Cov. in Mexican desert. Oecologia 92: 83-89.

Galeano AJR. 1993. Relación entre la fecha de cosecha, calidad fisiológica, sanitaria y longevidad en semilla de sorgo. Tesis de Maestría, Colegio de Postgraduados, México.

García HER.1994. Diferencias entre el frijol silvestre y domesticado en atributos morfofisiológicos y en su respuesta al almacenamiento inadecuado de la semilla. Tesis de Maestría, Colegio de Postgraduados, México.

Gómez-Pompa A,Vázquez C, Guevara S. 1972. The tropical rainforest: a nonrenovable resource. Science 177: 762-765. 
Lagunes ELC.1992. Efecto del frío sobre la faseolina y fracción 2 de las lobulinas de Phaseolus vulgaris L. Tesis de Maestría, Colegio de Postgraduados, México.

Larqué-Saavedra A, Rodríguez GMT. 1989. Evidences for maternal inheritance of abscisic acid in relation to drought in Zea mays. Phyton 49: 145-150.

Mendoza CMC. 1993. Algunos factores fisiológicos y anatómicos que afectan las relaciones fuente-demanda en maíz (Zea mays L.). Tesis de Maestría, Colegio de Postgraduados, México.

Montes GN.1992. Efecto de la defoliación sobre las relaciones hídricas y de fuente-demanda en sorgo sometido a déficit hídrico. Tesis de Maestría, Colegio de Postgraduados, México.

Montes UP. 1994. Actividad fotosintética y distribución de nutrimientos en zarzamora (Rubus spp.) en respuesta a la remoción de brotes vegetativos. Tesis de Maestría, Colegio de Postgraduados, México.

Moreno-Casasola P, Sánchez RG. 1990. La enseñanza de la ecología en México. Ciencias 4 (No. especial):96-111.

Orozco-Segovia A, Vázquez-Yanes C. 1989. Light effect on seed germination in Piper L. . Acta Oecologica Oecol. Plant. 10: 123-146.

Orozco-Segovia A, Vázquez-Yanes $\mathrm{C}$, Coates-Estrada R, PérezNasser N. 1987. Ecophysiological characteristics of the seed of the tropical forest pioneer Urera caracasana (Urticaceae). Tree Physiol. 3: 375-386.

Saenz QLA. 1994. Estudios sobre fisiología de la frigoconservación de tunas (Opuntia amyclaea T.) en relación a calidad y control de daños por frío. Tesis de Maestría, Colegio de Postgraduados, México.

Salinas MY. 1992. Acido abscísico, azúcares solubles y relaciones hídricas en inflorescencias de maíz sometido a sequía. Tesis de Maestría, Colegio de Postgraduados, México.

Sánchez CF.1994. Relaciones entre fuente y demanda en jitomate manejado con despuntes de población. Tesis de Maestría, Colegio de Postgraduados, México.

Sánchez F, Padilla JE, Pérez H, Lara,M. 1991. Control of Nodulin genes in root nodule development and metabolism. Ann. Rev. Plant Physiol. 42:507-528.

Sánchez JE. 1993. Two specific protein kinases from maize axes. Possible regulatory role in germination. 12th. Annual Missouri Plant Biochemistry, Molecular Biology and Physiology Symposium, 23-24.

Santos-Díaz MS. 1993. Obtención y caracterización de cultivos en suspensión de especies con diferente tolerancia al déficithídrico, y de líneas celulares de Capsicum annuum resistentes a polietilenglicol. Tesis de Doctorado. CINVESTAV. Irapuato, Gto. México.

Simpson J, Herrera-Estrella L. 1990. Ligh-regulated gene expression. Plant Sciences 9: 95-109.

Solarte CME. 1992. Efectos de estrés hídrico en la fisiología y actividades fotoquímicas de frijol. Tesis de Maestría, Colegio de Postgraduados, México.

Trejo CR. 1992. Caracterización in vitro de la tolerancia al déficit hídrico y a salinidad en células de Amaranthus hypochondriacus L. Tesis de Maestría, Colegio de Postgraduados, México.

Uscanga ME. 1992. Relaciones de interferencia inter e intraespecífica de Simsia amplexicaulis (Cav.) pers. y Zea mays L.: su efecto en algunos parámetros fisiológicos. Tesis de Maestría, Colegio de Postgraduados, México.

Vázquez GLM. 1994. Factores de defensa del clavel (Dianthusx caryophylluS L.) a la roya (Uromyces caryophyllinus (Sach) Wint.). Tesis de Doctorado, Colegio de Postgraduados, México.

Vázquez-Yanes C, Orozco-Segovia A. 1993. Pattern of seed longevity and germination in the tropical rainforest. Annu. Rev. Ecol. Syst. 24: 69-87. 